\title{
Commentary on "Enteral Lactoferrin Supplementation for Prevention of Sepsis and Necrotizing Enterocolitis in Preterm Infants"
}

\author{
Lauren Young $^{\mathrm{a}}$ William McGuire ${ }^{\mathrm{b}}$ Peter W. Fowlie \\ aDepartment of Neonatal Medicine, Evelina London Children's Hospital, St Thomas' Hospital, London, UK; \\ ${ }^{b}$ Centre for Reviews and Dissemination, University of York, York, UK; ' Department of Paediatrics, Ninewells Hospital \\ and Medical School, Dundee, UK
}

\section{Commentary}

Meta-analysis of outcome data from randomized controlled trials can increase the precision of estimates of the effect of interventions. These, however, need to be interpreted carefully as various forms of bias can inflate pooled effect size estimates. In particular, small study bias is recognised as an important limitation to the external validity and applicability of meta-analyses in systematic reviews [1]. Small trials have, on average, lower methodological quality than large trials. Typically, small trials are more likely to contain design limitations in the key domains: generation of random sequences, concealment of allocation, masking of parents, caregivers, and investigators, completeness of outcome assessment, and selectiveness of reporting [2]. Because of the prevalence of these sources of bias, small trials tend to have greater (though less precise) effect size estimates than large trials. Small study bias may, furthermore, be associated with (and be compounded by) "publication bias" - the tendency for reports of statistically significant or clinically important effects to be more likely than others to be submitted and accepted for publication [3]. Consequently, meta-analyses that contain data from many small trials can generate spuriously inflated effect size estimates.
Cochrane review authors attempt to identify this threat to the validity of meta-analyses by assessing statistical heterogeneity in the "forest plot" (the degree to which the effect size estimates from individual trials agree with each other beyond chance), and, where sufficient data are available, the symmetry of the "funnel plot" - the evenness of spread of the included trials' point estimates around the pooled estimate of effect. An asymmetric funnel plot, with an excess of data points from small trials favouring an intervention, indicates small study bias.

This phenomenon is evident in several Cochrane reviews of interventions for newborn infants including that of lactoferrin supplementation to prevent late-onset infection. Lactoferrin, a glycoprotein present in mammalian milk, has innate immunomodulatory and antimicrobial properties. Data from small randomized controlled trials (with 886 infant participants in total) had suggested that bovine lactoferrin supplementation of enteral feeds reduces late-onset infection in preterm infants by $>40 \%$. This finding prompted the conduct of new large trials of this intervention [4]. The recently updated Cochrane review incorporates data from these large trials, including one high-quality trial powered sufficiently to detect modest but important effects on the risk of late-onset infection [5]. 


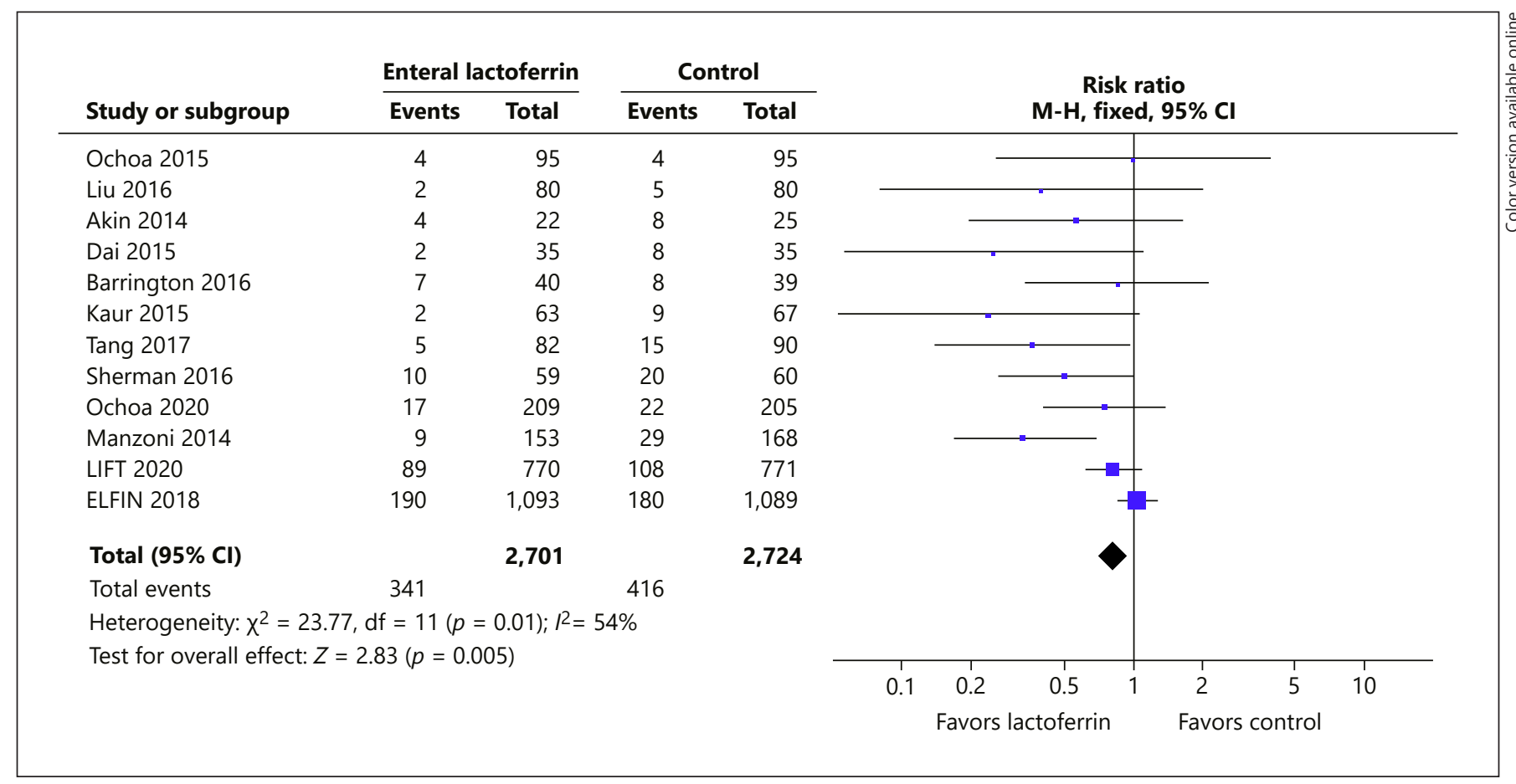

Fig. 1. Forest plot - lactoferrin supplementation versus placebo effect on late onset infection [7-18].

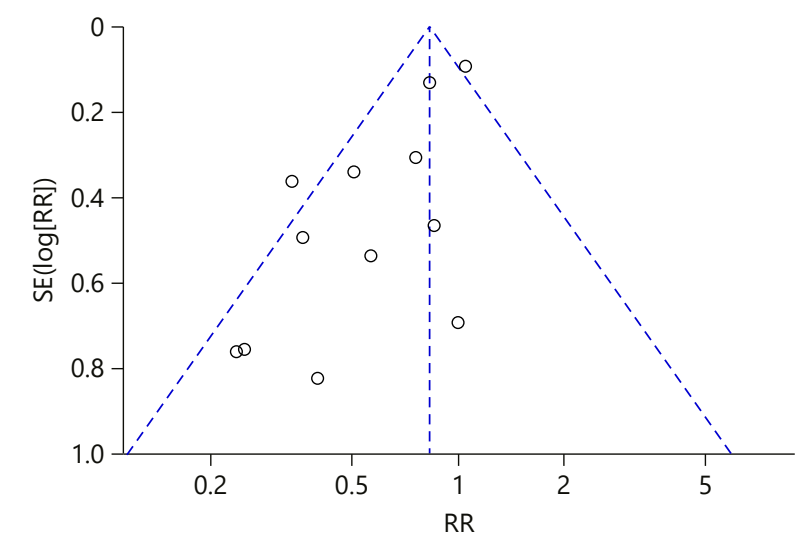

Fig. 2. Funnel plot - lactoferrin supplementation versus placebo effect on late onset infection.

The meta-analysis of data from all 12 included trials (with 5,425 infant participants) shows that lactoferrin supplementation is associated with a $17 \%$ reduction in the risk of late-onset infection: relative risk $0.83 ; 95 \%$ confidence interval 0.72-0.94 (Fig. 1). The forest plot, however, indicates statistical heterogeneity in the meta-anal- ysis $\left(I^{2}=54 \%\right)$, and the funnel plot is markedly asymmetric consistent with small study or publication bias (Fig. 2). While the large trials do not show evidence of an effect, the small trials appear to have skewed the pooled effect size estimate in favour of the intervention. The review authors factored in this concern in evaluating the evidence using the Grades of Recommendation, Assessment, Development, and Evaluation (GRADE) method [6]. Therefore, although the pooled estimate of effect suggests that lactoferrin may reduce the risk of late-onset infection, the evidence is assessed as "low certainty."

The research community internationally has made considerable efforts to address the threats posed by small studies and publication bias to the integrity of evidence syntheses. Foremost amongst these has been the requirement for the methodological details of trials (including proposed sample size and primary outcomes) to be registered prospectively in clinical trial databases [1]. Cochrane review authors can search trial registries (e.g., via the US National Library of Medicine's clinicaltrials.gov/) for unreported trials. The success of this and related initiatives in combatting publication bias and other forms of small study bias remains unclear, however, and further measures, including the exclusion of unregistered trials from Cochrane reviews, have been advocated [19]. 


\section{Acknowledgement}

Cochrane Neonatal Reviews are produced with support from Vermont Oxford Network, a worldwide collaboration of health professionals, dedicated to providing evidence-based care of the highest quality for newborn infants and their families.

\section{Conflict of Interest Statement}

William McGuire is the co-coordinating editor of Cochrane Neonatal and the chief investigator of the ELFIN trial.

\section{Funding Sources}

National Institute of Health Research (UK) Cochrane Programme Grant (16/114/03).

\section{Author Contributions}

All authors contributed to this commentary.

\section{References}

1 Gale C, McGuire W, Juszczak E. Randomised controlled trials for informing perinatal care. Neonatology. 2020;117(1):8-14.

2 Lai NM, Ong JMJ, Chen KH, Chaiyakunapruk N, Ovelman C, Soll R. Are neonatal trials better conducted and reported over the last 6 decades? An analysis on their risk-ofbias status in Cochrane reviews. Neonatology. 2019;116(2):123-31.

3 Kirpalani H. Control of bias has been recognized as important for a long time - but are we finally in control? Neonatology. 2019; 116(2):185-7.

4 Walsh V, McGuire W. Immunonutrition for preterm infants. Neonatology. 2019;115(4): 398-405.

5 Pammi M, Suresh G. Enteral lactoferrin supplementation for prevention of sepsis and necrotizing enterocolitis in preterm infants. Cochrane Database Syst Rev. 2020;6(3): CD007137.

6 Guyatt GH, Oxman AD, Vist GE, Kunz R, Falck-Ytter Y, Alonso-Coello P, et al. GRADE: an emerging consensus on rating quality of evidence and strength of recommendations. BMJ. 2008;336(7650):924-6.

7 Ochoa TJ, Zegarra J, Cam L, Llanos R, Pezo A, Cruz K, et al. Randomized controlled trial of lactoferrin for prevention of sepsis in Peruvian neonates less than $2500 \mathrm{~g}$. Pediatr Infect Dis J. 2015;34(6):571-6.
8 Liu YH, Guan HS, Liang GJ, Li YZ, Zuo A. The effect of lactoferrin on low birth weight neonates during hospitalization. Matern Child Health Care China. 2016;31(21):44645.

9 Akin IM, Atasay B, Dogu F, Okulu E, Arsan S, Karatas HD, et al. Oral lactoferrin to prevent nosocomial sepsis and necrotizing enterocolitis of premature neonates and effect on $\mathrm{T}$ regulatory cells. Am J Perinatol. 2014;31(12): 1111-20.

10 Dai JZ, Xie C. The effect of lactoferrin supplementation combining Lactobacillus rhamnosus for prevention of late onset sepsis in premature neonates. China Pract Med. 2015;10: 98-100.

11 Barrington KJ, Assaad MA, Janvier A. The Lacuna Trial: a double-blind randomized controlled pilot trial of lactoferrin supplementation in the very preterm infant. J Perinatol. 2016;36(8):666-9.

12 Kaur G, Gathwala G. Efficacy of bovine lactoferrin supplementation in preventing late-onset sepsis in low birth weight neonates: a randomized placebo-controlled clinical trial. J Trop Pediatr. 2015;61(5):370-6.

13 Tang JP, Sun HQ, Zheng YH, Lu GH. Randomized control trial of lactoferrin for the prevention of late onset sepsis in premature neonates. Matern Child Health Care China. 2017;32(6):1223-5.
14 Sherman MP, Adamkin DH, Niklas V, Radmacher P, Sherman J, Wertheimer F, et al. Randomized controlled trial of talactoferrin oral solution in preterm infants. J Pediatr. 2016;175:68-73.e3

15 Ochoa TJ, Zegarra J, Bellomo S, Carcamo CP, Cam L, Castañeda A, et al. Randomized controlled trial of bovine lactoferrin for prevention of sepsis and neurodevelopment impairment in infants weighing less than 2000 grams. J Pediatr. 2020;219:118-25.e5.

16 Manzoni P, Meyer M, Stolfi I, Rinaldi M, Cattani S, Pugni L, et al. Bovine lactoferrin supplementation for prevention of necrotizing enterocolitis in very-low-birth-weight neonates: a randomized clinical trial. Early Hum Dev. 2014;90(Suppl 1):S60-5.

17 Tarnow-Mordi WO, Abdel-Latif ME, Martin A, Pammi M, Robledo K, Manzoni P, et al. The effect of lactoferrin supplementation on death or major morbidity in very low birthweight infants (LIFT): a multicentre, doubleblind, randomised controlled trial. Lancet Child Adolesc Health. 2020;4(6):444-54.

18 ELFIN trial investigators group. Enteral lactoferrin supplementation for very preterm infants: a randomised placebo-controlled trial. Lancet. 2019;393(10170):423-33.

19 Roberts I, Ker K, Edwards P, Beecher D, Manno D, Sydenham E. The knowledge system underpinning healthcare is not fit for purpose and must change. BMJ. 2015;350:h2463. 


\section{Cochrane Abstract}

Background: Lactoferrin, a normal component of human colostrum and milk, can enhance host defenses and may be effective for prevention of sepsis and necrotizing enterocolitis (NEC) in preterm neonates. Objectives: The objective of this study was to assess the safety and effectiveness of lactoferrin supplementation to enteral feeds for prevention of sepsis and NEC in preterm neonates. Secondarily, we assessed the effects of lactoferrin supplementation to enteral feeds on the duration of positive-pressure ventilation, development of chronic lung disease (CLD) or periventricular leukomalacia, length of hospital stay to discharge among survivors, and adverse neurological outcomes at 2 years of age or later. Search Methods: We used the standard search strategy of Cochrane Neonatal to update our search. We searched the Cochrane Central Register of Controlled Trials (CENTRAL 2019, Issue 9), MEDLINE via PubMed (1966 to January 20, 2020), PREMEDLINE (1996 to January 20, 2020), Embase (1980 to January 20, 2020), and CINAHL (1982 to January 20, 2020). We also searched clinical trials databases, conference proceedings, and the reference lists of retrieved articles for randomized controlled trials and quasi-randomized trials. Selection Criteria: In our search, we included randomized controlled trials (RCTs) evaluating enteral lactoferrin supplementation at any dose or duration to prevent sepsis or NEC in preterm neonates. Data Collection and Analysis: We used the standard methods of Cochrane Neonatal and the GRADE approach to assess the certainty of evidence. Main Results: Meta-analysis of data from 12 randomized controlled trials showed that lactoferrin supplementation to enteral feeds decreased suspected or confirmed late-onset sepsis (typical RR 0.80, 95\% Cl 0.72-0.89; typical RD -0.05 , $95 \% \mathrm{Cl},-0.07$ to -0.02 ; NNTB $20,95 \% \mathrm{Cl} 14-50 ; 12$ studies, 5,425 participants, low-certainty evidence) and decreased length of hospital stay (MD -2.38 to $95 \% \mathrm{Cl},-4.67$ to -0.09 ; 3 studies, 1,079 participants, low-certainty evidence). A subgroup analysis including data of infants with confirmed sepsis demonstrates a decrease in confirmed late-onset sepsis (typical RR 0.83, 95\% Cl 0.73-0.94; typical RD $-0.03,95 \% \mathrm{Cl}$, -0.04 to -0.01 ; NNTB $33,95 \% \mathrm{Cl} 25-100 ; 12$ studies, 5,425 participants, low-certainty evidence). Sensitivity analysis including only good methodological certainty studies sug- gested a decrease in late-onset sepsis (both suspected and confirmed) with enteral lactoferrin supplementation (typical RR $0.82,95 \% \mathrm{Cl}, 0.74-0.91$; typical RD $-0.04,95 \% \mathrm{Cl},-0.06$ to -0.02 ; NNTB 20, 95\% Cl 14-50; 9 studies, 4,702 participants, low-certainty evidence). There were no differences in NEC stage II or III (typical RR 1.10, $95 \% \mathrm{Cl}, 0.86-1.41$; typical RD $-0.00,95 \% \mathrm{Cl},-0.02$ to $0.01 ; 7$ studies, 4,874 participants; low-certainty evidence) or "all-cause mortality" (typical RR $0.90,95 \% \mathrm{Cl} 0.69-1.17$; typical RD $-0.00,95 \% \mathrm{Cl},-0.01$ to $0.01 ; 11$ studies, 5,510 participants; moderate-certainty evidence). One study reported no differences in neurodevelopmental testing by Mullen's or Bayley III at 24 months of age after enteral lactoferrin supplementation (1 study, 292 participants, low-certainty evidence). Lactoferrin supplementation to enteral feeds with probiotics decreased late-onset sepsis (RR $0.25,95 \% \mathrm{Cl} 0.14-0.46 ; \mathrm{RD}-0.13,95 \% \mathrm{Cl}-0.18$ to -0.08 ; NNTB 8, 95\% Cl 6-13; 3 studies, 564 participants; lowcertainty evidence) and NEC stage II or III (RR $0.04,95 \% \mathrm{CI}$ $0.00-0.62 ; \mathrm{RD}-0.05,95 \% \mathrm{Cl}-0.08$ to -0.03 ; NNTB $20,95 \% \mathrm{Cl}$ 12.5-33.3; 1 study, 496 participants; very low-certainty evidence), but not "all-cause mortality" (very low-certainty evidence). Lactoferrin supplementation to enteral feeds with or without probiotics had no effect on CLD, duration of mechanical ventilation, or threshold retinopathy of prematurity (low-certainty evidence). Investigators reported no adverse effects in the included studies. Authors' Conclusions: We found low-certainty evidence from studies of good methodological quality that lactoferrin supplementation of enteral feeds decreases late-onset sepsis (both suspected and confirmed, and confirmed only) but not NEC $\geq$ stage II or "all cause mortality" or neurodevelopmental outcomes at 24 months of age in preterm infants without adverse effects. Low- to very low-certainty evidence suggests that lactoferrin supplementation of enteral feeds in combination with probiotics decreases late-onset sepsis (data from confirmed sepsis only) and NEC $\geq$ stage II in preterm infants without adverse effects; however, there were few included studies of poor methodological quality. The presence of publication bias and small studies of poor methodology that may inflate the effect size make recommendations for clinical practice difficult. 